\title{
VIBRATORY MOVEMENT IN THE CYTOPLASM OF ERYTHROCYTES
}

\author{
BY \\ R. J. V. PULVERTAFT \\ From the Westminster Hospital School of Medicine
}

(RECEIVED FOR PUBLICATION, MAY 26, 1949)

In 1890 Browicz described an intracellular vibratory movement in human erythrocytes, which he believed to be a pathological condition, from four cases of anaemia. It was found in one case of pernicious anaemia, and was stated to disappear following a remission; it was also found in two cases of malignant disease. He ascribed it to abnormal reactions between pathological erythrocytes and their plasma. In his view it was not a vital phenomenon since it persisted at room temperature when leucocytic activity had subsided, and he stated that it continued for at least 24 hours.

Cabot (1901) made several references to this motion. He stated :

"If we focus carefully on a red cell we can usually make out a fine wavy so-called molecular motion in it. This is quite different from the active amoeboid movement observed in dying cells, and from the rapid dancing of malarial pigment."

In modern textbooks of cytology and haematology no reference to this motion is found. On the contrary, the absence of structure as seen by all methods of microscopy except the polariscope is stressed. Yet motion is not possible in the absence of structure.

During the examination of avian and amphibian erythrocytes by phase contrast illumination a rapid and regular oscillatory movement was easily noted in the cytoplasm. The cytoplasm of normal and abnormal erythrocytes revealed the same motion, and indeed all erythrocytes examined, including those of goose, chicken embryo, hen, rabbit, guinea-pig, and mouse, show it clearly. It is best seen in the large nucleated erythrocytes of birds and frogs.

"Brownian movement" is the oscillation of visible particulate material when suspended in fluid. The vibratory phenomenon here discussed does not involve motion in particulate material within the limits of resolution of the microscope. No such particulate material exists in the erythro- cyte. The phenomenon consists of a rapidly alternating change of the refractive index of the cytoplasm, leading to an appearance of alternate lightening and darkening of certain areas. These areas are, roughly speaking, arranged radially, and are exceedingly close together, so that the whole of the content gives the appearance of intense and continuous agitation.

The cell membrane of mammalian erythrocytes, when examined under a cover-slip pressed firmly down, shows a continuous irregular deformation of outline, which can be clearly seen by dark ground illumination; by careful focusing it can be noted that the upper surface, as well as the edge, is in continuous motion. No such motion can be seen with the erythrocytes of bird or frog; the latter indeed have, in the cases examined, on their surface minute granules which remain motionless. It follows therefore that the apparent vibration in the cytoplasm is not due to a change in refractive index of the contents as the cell varies locally in thickness owing to movement of the cell membrane, but to a periodic change of some kind in the cell contents. As will be related, this vibration can be inhibited by sodium fluoride.

Since the geometric form of erythrocytes is conditioned by the physical nature of their environment, it is necessary to adopt a special technique to observe the vibratory motion. In saline or in Tyrode's solution mammalian erythrocytes assume a spherical form ; this form is also assumed with many reagents. Blood must therefore be collected in minimal amounts of $3 \%$ sodium citrate, or examined fresh and undiluted.

When crenated, erythrocytes show poor vibration ; when spherical, they show none, and indeed they appear to be dense and compact. The best condition for viewing cells is obtained by placing a small drop on a slide, and pressing the coverslip very firmly down under blotting-paper. 
When examined by normal transmitted light, vibratory movement can sometimes be seen if the iris diaphragm is well stopped down, but it is most difficult to observe. By dark ground illumination it is very clearly seen in avian cells, but in mammalian cells it is in part obscured by the motion of the cell membrane. It is best observed by phase contrast. The illuminant for phase contrast microscopy is most important, especially with high power objectives. After experimenting with many types, the best was found to be the B.T.H. Mazda

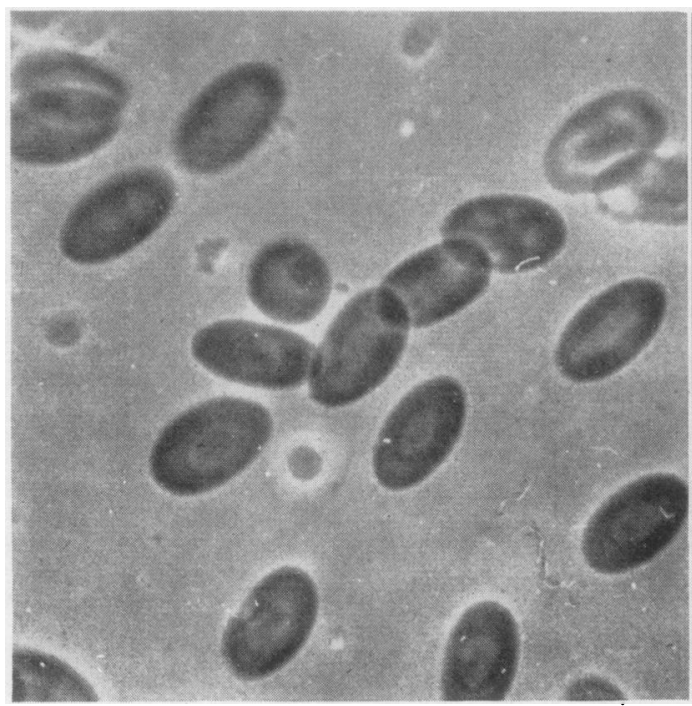

FIG. 1.-Avian erythrocytes in plasma. Phase contrast $\times 900$.

mercury vapour electric discharge lamp, box type ME $250 \mathrm{~W} / 50 / 5$, as recommended by Clegg and Foster-Carter (1946).

\section{Experiments}

Duration and Period of Vibration.-Vibration continues unaltered up to 48 hours in many cases. In general it appears to persist until haemolysis sets in. The oscillatory movement appears to be of the same periodic order as that of minute particles suspended in the plasma. Attempts to measure the period with a stroboscope were not successful.

Temperature.-No change in vibration was observed between $0^{\circ} \mathrm{C}$. and $37^{\circ} \mathrm{C}$. When further warmed, haemolysis sets in, and vibration ceases to be observable.

Haemolysis. - In whatever way haemolysis is produced-i.e., by the action of complement on sensitized cells-vibration gradually ceases. "Ghost" erythrocytes show no trace of vibration, but they do frequently contain minute granules in Brownian movement.

Vibration in Other Cells. - Nothing resembling this has been noted during long examination of other mammalian cells, or in the cells of lower forms of life-e.g., Daphnia. On a warm stage the granules in many cells oscillate, but this appearance is of an entirely different order from the comprehensive erythrocytic vibration.

Reticulum in unstained reticulocytes cannot be resolved by phase contrast, but blood with $30 \%$ of reticulocytes showed vibration in all the erythrocytes.

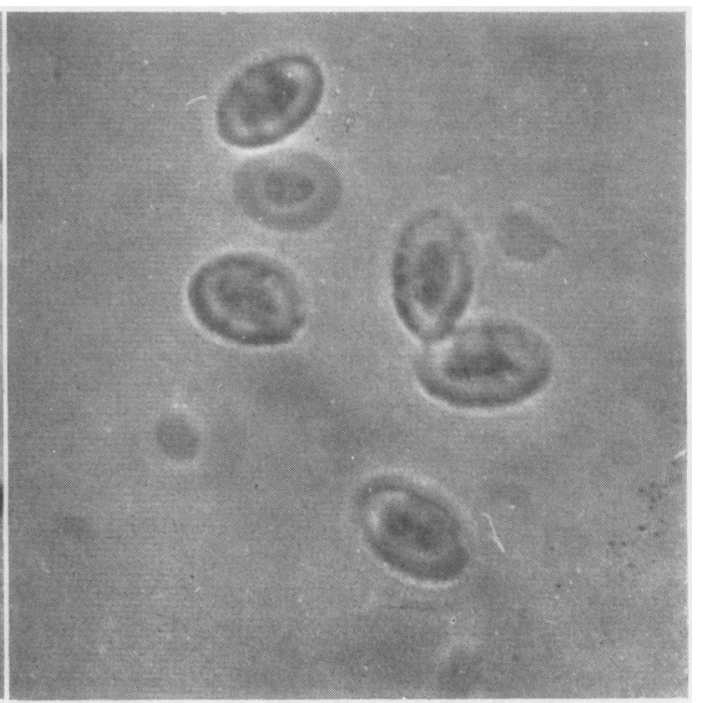

F.G. 2.-Avian erythrocytes in plasma with $0 \cdot 1 \mathrm{ml}$. M sodium fluoride added per ml. plasma. Phase contrast $\times 900$.

When vitally stained by cresyl blue the reticulocytes are identifiable; the reticulum remains motionless but the cytoplasmic vibration continues.

The abnormally shaped erythrocytes seen in anaemia also show vibration. It is visible in erythroblasts but not in pro-erythroblasts either from bone marrow or in the peripheral circulation. Not until a halo is visible around the nucleus by phase contrast is vibration visible; this halo is not seen in pro-erythroblasts or in more primitive cells of the series.

Effect of Gases.-Vibration continues in cells which have been exposed to an atmosphere of pure oxygen, nitrogen, carbon dioxide, and carbon monoxide.

Effect of Certain Chemical Reagents.-Vibration ceases immediately with formalin at a concentration of 1 in 2,000 of the $40 \%$ solution.

The effect of sodium fluoride is noteworthy. When $0.1 \mathrm{ml}$. of a molar solution is added to $0.9 \mathrm{ml}$. of citrated blood, there is no immediate change in the appearances, but within three minutes vibration ceases 
with mammalian erythrocytes. Avian erythrocytes also cease to show vibration, but in addition show another change. By phase contrast there is normally no halo around the nucleus, but after the addition of sodium fluoride a marked halo develops, and the cytoplasmic contents appear to be withdrawn and condensed towards the periphery. If avian cells treated with sodium fluoride are fixed and stained by Leishman's stain, they are, however, indistinguishable from normal cells.

\section{Discussion}

The first observers of this motion accepted it as " molecular movement," and apparently saw it by transmitted light. They worked in an era of scrupulous microscopical observation of living cells; to-day we are rather limited by the artifacts of fixation and staining. It certainly is not an artifact produced by the alternation of current to an A.C. lamp, since it is equally well seen with a D.C. arc. A reason has been given for excluding the suggestion that the apparent motion is due to oscillations in the cell membrane ; indeed by phase contrast the impression given is definitely one of turbulence in the cytoplasm.

Solutions of haemoglobin in distilled water or in gelatin show no trace of the motion, and ghost erythrocytes equally show no motion. Either the haemoglobin when inside the cell itself occasions the motion, or it acts as an indicator, making motion visible. This might, for example, be the case if it were periodically subject to focal concentration, with a change of refractive index. It is not possible to resolve any particulate matter in erythrocytes by any microscopical technique; the haemoglobin molecule is well outside the limits of resolution.

The main function of haemoglobin is the acceptance and transference of oxygen. The motion, however, cannot be related to this, since when saturated with carbon monoxide the cell still vibrates. It also vibrates when sodium hydrosulphite is added, until haemolysis sets in.

When the cell is "fixed" by formalin vibration ceases, doubtless owing to physical alteration in the colloidal contents. The action of sodium fluoride is of greater interest, particularly since it appears to produce an alteration of the distribution of haemoglobin in the cell, at least in avian erythrocytes.

Whereas many reagents added to suspensions of erythrocytes in plasma tend to produce changes in the geometrical form of erythrocytes, sodium fluoride in the strength used does not; its effect is only noted optically by the cessation of vibratory motion in the mammalian erythrocyte, and redistribution of haemoglobin in the avian. Wilbrandt (1940) has shown that sodium fluoride causes permeability to cations in erythrocytes, and this is said to be related to interruption of glycolysis. The normal erythrocyte is impermeable to potassium ; this characteristic is altered by sodium fluoride. So far as the experiments related here give any indication of the nature of the vibratory motion, they suggest that it is related to glycolysis. The vibratory motion itself is an indication that the refractive index of the cytoplasmic contents is constantly changing; the alternation of dark and light seen by phase contrast is probably due to changing concentrations of the haemoglobin, and this may be related to metabolism, and particularly to glycolysis.

\section{Summary}

Mammalian, avian, and amphibian erythrocytes show a vibratory motion in the cytoplasm, best seen by phase contrast. No other cell of many examined, whether from mammals or from lower forms of life, exhibits this motion. It is seen in reticulocytes and erythroblasts, but not in proerythroblasts. It is not seen in de-haemoglobinized erythrocytes or in a solution of haemoglobin. It is inhibited by sodium fluoride, which produces a redistribution of haemoglobin in avian erythrocytes. The possible relation to glycolytic metabolism is discussed.

\section{REFERENCES}

Browicz (1890). Centr. f. d. med. Wissen, 28, 625.

Cabot, R. C. (1901). A Guide to the Clinical Examination of the Blood, p. 52. 4th ed. Longmans, Green \& Co. London.

Clegg, J. W., and Foster-Carter, A. F. (1946). Brit. J. Tuberc., 40, 98. Wilbrandt, W. (1940). Pflüg. Arch. ges. Physiol., 243, 519. 